\title{
Telephone Counseling for Smoking Cessation: Effects of Single-Session and Multiple-Session Interventions
}

\author{
Shu-Hong Zhu, Vincent Stretch, Mark Balabanis, Bradley Rosbrook, Georgia Sadler, and John P. Pierce \\ University of California, San Diego
}

\begin{abstract}
Smokers $(N=3,030)$ were randomized to receive 1 of 3 interventions: (a) a self-help quit kit, (b) a quit kit plus 1 telephone counseling session, or (c) a quit kit plus up to 6 telephone counseling sessions, scheduled according to relapse probability. Both counseling groups achieved significantly higher abstinence rates than the self-help group. The rates for having quit for at least 12 months by intention to treat were $5.4 \%$ for self-help, $7.5 \%$ for single counseling, and $9.9 \%$ for multiple counseling. The 12-month continuous abstinence rates for those who made a quit attempt were $14.7 \%$ for self-help, $19.8 \%$ for single counseling, and $26.7 \%$ for multiple counseling. A dose-response relation was observed, as multiple sessions produced significantly higher abstinence rates than a single session. The first week after quitting seems to be the critical period for intervention.
\end{abstract}

Telephone counseling has attracted increasing interest as an alternative system for delivery of services in the field of smoking cessation (e.g., Anderson, Duffy, Hallet, \& Marcus, 1992; Curry, McBride, Louie, Grothaus, \& Wagner, 1992; DeBusk et al., 1994; Lando, Hellerstedt, Pirie, \& McGovern, 1992; Orleans et al., 1991; Ossip-Klein et al., 1991; Prochaska, DiClemente, Velicer, \& Rossi, 1993; Shiffman, Read, Maltese, Rapkin, \& Jarvik, 1985). From the smoker's standpoint, its main attractions are accessibility and convenience. There are no transportation difficulties and fewer scheduling conflicts than in most other cessation programs. Also, receiving counseling in the privacy of one's home provides treatment access to individuals who might not normally seek "counseling" to quit smoking. These factors encourage smokers to use the service ( $Z$ hu et al., 1995).

Shu-Hong Zhu, Vincent Stretch, Mark Balabanis, Bradley Rosbrook, Georgia Sadler, and John P. Pierce, Cancer Center, University of California, San Diego.

Part of this study was presented in March 1993 at the 14th Scientific Conference of the Society of Behavioral Medicine in San Francisco.

This study was supported by funds received from the Tobacco Tax Health Protection Act of 1989, Proposition 99, under Contract Number 90-10961 with the California Department of Health Services. We thank project officer April Roesler for her help throughout the study. The analysis was facilitated through Grant 92-15416 from the same agency.

We thank Eva Martinez and Mary Lou Munguía for their contributions to the early development of the study and Jacqueline Estey and Judy Quan for managing the data. Others who worked on the project's counseling and evaluation teams are Doraine Bell, Kim Bloom, Deidra Ellis, Denise Freeman, Fernando Martínez, Melissa McCool, Denise Scott, Gary Tedeschi, Esperanza Villegas, and Eniko Voss. Christopher Anderson, Norman Anderson, Charles Berry, Elizabeth Gilpin, Edward Lichtenstein, and William Miller provided many helpful comments on an early version of the article.

Correspondence concerning this article should be addressed to ShuHong Zhu, Cancer Center, University of California, San Diego, Mail Code 0901, 9500 Gilman Drive, La Jolla, California 92093-0901. Electronic mail may be sent via Internet to szhu@ucsd.edu.
Most studies on telephone counseling for smoking cessation have focused on the question of whether a brief intervention over the telephone can significantly increase the success rate for quitting smoking. The interventions that have been used vary in two respects: (a) whether the counseling calls are initiated by the counselor or by the client (proactive vs. reactive) and (b) the number of sessions provided (ranging from one to four). The results are somewhat mixed. While several studies have shown that such an intervention significantly increases the success rate (e.g., Orleans et al., 1991; Ossip-Klein et al., 1991), others report only a short-term effect, with the long-term outlook no better than that of selfhelp (e.g., Curry et al., 1992; Lando et al., 1992). Those studies that have shown a significant intervention effect for telephone counseling, however, did not include a randomized design to test for a dose-response relation between the number of sessions and the treatment effect.

The present study examined the effects of two levels of telephone counseling and compared them with the effects of a selfhelp approach. The lower intensity counseling consisted of one session before quitting. The higher intensity counseling included the same pre-quit session plus up to five sessions after the smoker had quit. We tested two hypotheses: (a) that counseling would produce a higher abstinence rate than a self-help quit kit, and (b) that multiple sessions of counseling would produce a higher abstinence rate than a single session.

The design of the telephone interventions used in this study was based on the following principles. First, substantial counseling effort should be spent on promotion of the client's motivation to change, a basic element of effective brief interventions for addictive behaviors (Miller \& Rollnick, 1991). Second, the counselor should help the client develop effective coping strategies to overcome the urge to smoke (Shiffman, 1982). Third, follow-up sessions geared toward relapse prevention should be as timely as possible to maximize their effect (Zhu, 1993). This last point is a significant feature of the multiple counseling protocol in the present study and is explained later. 


\section{Three Features of the UCSD Telephone Counseling Program}

The University of California, San Diego (UCSD), telephone counseling program had three distinctive features: proactive counseling, a structured counseling protocol, and relapse-sensitive scheduling (Zhu, 1993).

\section{Proactive Counseling}

The UCSD Smokers' Helpline adopted a proactive counseling procedure; that is, once a smoker took the first step by calling for help, all subsequent sessions were initiated by the counselor. Calls were never made without the previous consent of the client, who retained the option of deciding later not to receive them. The proactive procedure is a form of "active helping" (Miller \& Rollnick, 1991). It engenders good rapport, provides an opportunity to arrest faltering motivation, and reduces the attrition rate.

\section{Structured Counseling Protocol}

The UCSD Smokers' Helpline developed a structured counseling protocol with the following goals in mind. The first was to keep the counseling brief and focused by furnishing counselors with the essential ingredients of brief intervention (e.g., Miller \& Rollnick, 1991). The second was to provide a counseling intervention that was sensitive to the different phases of the quitting process. Accordingly, the helpline developed a protocol containing six separate but related questionnaires to be used in sequence for up to six sessions, one before quitting and five afterward. The counseling session that occurred before smokers made a quit attempt focused on promoting the motivation to change, whereas the postquit counseling sessions focused on relapse prevention.

In the first session, before the quit attempt, smokers were first assessed on their smoking history, quitting history, and confidence about the impending quit attempt. Their desire to change and related fears (ambivalence characteristic of addictive behavior change) were acknowledged, discussed, and clarified. A significant portion of the counseling was then spent promoting smokers' self-efficacy for a successful attempt. This included asking smokers to identify the situations in which it would be most difficult to refrain from smoking once they had quit and to plan realistic strategies for coping with those situations. This was done in an effort to break down the seemingly overwhelming task of quitting into manageable components. This final portion of the first counseling session dealt with relapse prevention as well as motivation. The entire pre-quit session usually lasted about $50 \mathrm{~min}$.

The follow-up sessions, for those who received multiple counseling, occurred after the quit attempt and focused on relapse prevention. First, clients were asked whether their anticipated difficult situations were indeed problematic. Previously formulated coping strategies were reviewed, and their efficacy was assessed. The emphasis at this point was on either the successful implementation of effective coping strategies or the development of new strategies to replace or augment those that once seemed promising but were subsequently found to be in- effective. Second, clients were encouraged to change their selfimage as a smoker to that of a nonsmoker, a concept that was given increasing weight in the final sessions ( $\mathrm{Zhu}, 1993$ ). Although the latter stage of counseling was primarily concerned with relapse prevention, counselors often revisited the issue of motivation in these calls as well. ${ }^{1}$

Each of the five follow-up sessions took about $20 \mathrm{~min}$. Therefore, if the client received all six sessions, the total counseling time would be under $3 \mathrm{hr}$. The time spent counseling was not long but was used judiciously as explained later.

\section{Relapse-Sensitive Scheduling of Multiple Follow-Up Sessions}

In contrast to the traditional schedule of weekly sessions, the helpline adopted a new scheduling method that arranged follow-up sessions according to the probability of relapse ( $\mathrm{Zhu} \&$ Pierce, 1995). A typical relapse curve takes the shape of a negatively accelerated function, as illustrated in Figure 1. The probability of relapse is much greater in the initial stage than in the later stages of quitting. In other words, smokers' need for help is strongest soon after they attempt to quit. Therefore, to optimize the effect of counseling, the helpline scheduled calls in a logarithmic manner rather than at equal intervals. The vertical dashed lines connected to the horizontal axis in Figure 1 indicate the timing of the five follow-up sessions: They occurred $1,3,7,14$, and 30 days after the quit attempt. Such a probabilistic arrangement has been called a relapse-sensitive schedule (Zhu \& Pierce, in press).

\section{Method}

\section{Subjects}

Subjects were recruited from San Diego County mainly by including the helpline's toll-free telephone numbers in advertisements of the county's ongoing antismoking campaign. Smokers who called were screened for eligibility using three criteria: (a) readiness to quit within a week of first contacting the helpline, (b) readiness of any other smokers living in the same household to quit also, and (c) absence of more serious problems (e.g., current psychiatric conditions). Over a 17 month recruitment period, 3,030 smokers met these criteria. Of that number, $57 \%$ were female; $40 \%$ had fewer than 12 years of education; $81 \%$ were White, $8 \%$ were Black, $7 \%$ were Hispanic, $2 \%$ were Asian or Pacific lslander, and 2\% were of other ethnic-racial backgrounds. The mean age of the subjects was 36.3 years $(S D=12.2)$. The mean number of cigarettes smoked per day was $20.2(S D=10.4)$, and the average number of years the subjects had smoked was $19.5(S D=11.8)$.

\section{Design}

Subjects were randomized into three groups. (a) The self-help group received a self-help quit kit on how to quit smoking, which included either $A$ Lifetime of Freedom From Smoking by the American Lung Association or The Cool Turkey Quitting Guide by the Stanford Center for Research in Disease Prevention. The self-help group, despite the minimal intervention they received, served as a control group in this study. (b) The single counseling group received the same self-help ma-

\footnotetext{
${ }^{1}$ Training for the counseling protocol is available from Shu-Hong
} 


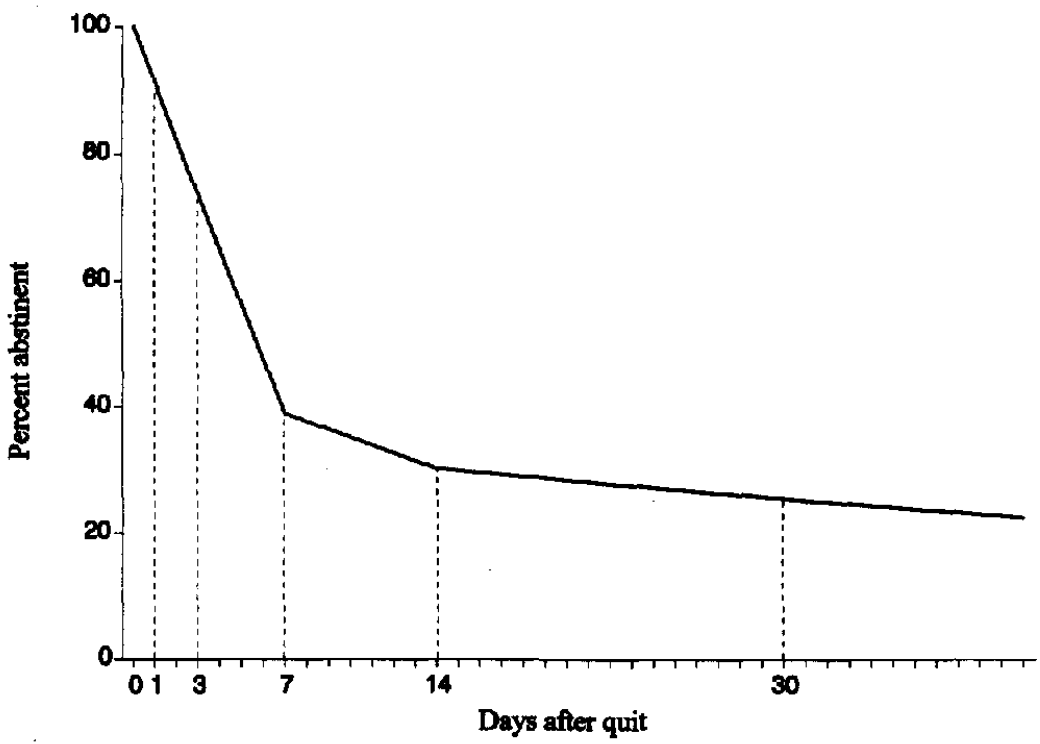

Figure 1. Relapse-sensitive scheduling of multiple counseling sessions.

terials and a 50-min pre-quit session of counseling. (c) The multiple counseling group received the same self-help materials, the pre-quit counseling session, and five follow-up sessions.

The subjects were randomized at the time of screening by the last two digits of their phone numbers. Those whose last two digits were from 00 to 29 were assigned to the self-help group. The rest ( $30-99$ ) were divided between the two counseling groups according to whether the final digit was odd or even. Care was also taken to ensure that each counselor was assigned a comparable number of clients in both single and multiple counseling. Recruitment of subjects occurred from February 1, 1991 to June 30,1992 . Of those randomized over this period, 841 subjects were assigned to the self-help group, 1,143 to the single counseling group, and 1,046 to the multiple counseling group.

\section{Counselors}

Over the course of the study, 10 paid counselors worked on the project. Two of them were finishing graduate degrees in counseling psychology. The other eight were lay counselors. All counselors received about $45 \mathrm{hr}$ of training with respect to the program protocol before they started counseling.

\section{Evaluation Procedure}

A separate group of evaluators, independent of the counseling staff, conducted up to four follow-up interviews per subject. For the self-help and single counseling groups, these interviews occurred at approximately $2,4,7$, and 13 months from the date subjects were screened and entered the experiment. For the multiple counseling group, only the latter three interviews were sought because many of these subjects were still being counseled at 2 months. All participants were scheduled to receive both the first (2-or 4-month) and the last (13-month) evaluation interviews. Each intermediate interview, however, was scheduled only for those who reported not smoking at the previous evaluation interview. For any one scheduled evaluation call, the interviewers made 25 attempts to contact each subject, at various times of the day and on different days of the week, before classifying the subject as a noncontact.

\section{Measures}

Abstinence rate by intention to treat. Percentages were calculated of the subjects who were abstinent for given lengths of time during the 12 month period after screening. The lengths of abstinence considered were 1 week, 1 month, 3,6, and 12 months. If a subject made more than one quit attempt, the longest quit attempt was used in the analysis. Those who did not receive the complete set of evaluations were analyzed using the data from those evaluations that they did receive. Those whom the helpline could not contact at all were treated as never having made any quit attempt.

The total percentage of subjects who could not be contacted for follow-up evaluations was $14.3 \%$ (14.3\% for self-help, $16.6 \%$ for single counseling, and $12.3 \%$ for multiple counseling), with $11.4 \%$ having had their phone disconnected or having moved and left no forwarding number and $2.9 \%$ unavailable after 25 attempts or having declined the follow-up interviews.

The next two measures assessed the effect of counseling by focusing on the quit attempts that were initiated during the first 3 months after screening. Three months was the longest time that a subject would be in contact with a counselor. This period takes into account the time needed for self-help materials to arrive, the lag time between the first session and the quit date, and time lost to early failed quit attempts. For either measure, any quit attempts initiated after the first 3 months were not attributed to counseling.

Twenty-four-hour quit rate in the first 3 months. For subjects in each group, percentages were calculated for those quit attempts that lasted at least $24 \mathrm{hr}$ within the period of counseling. A difference in 24-hr quit rates between the self-help group and the two counseling groups indicates the impact of counseling on whether smokers made a serious attempt to quit.

Duration of abstinence for quit attempts initiated in the first 3 months. A survival analysis of quit attempts made within the period of counseling estimates the percentage of subjects remaining abstinent over time, or an "abstinence curve." Such curves are paradoxically called relapse curves in the addiction literature (U.S. Department of Health and Human Services, 1988). Relapse is defined here as a return to smoking for more than 1 day after any period of abstinence. If subjects made more 
than one quit attempt in the first 3 months, the most recent one was used in the analysis. A comparison of relapse curves for quit attempts initiated in the first 3 months provides a clear picture of the relapseprevention effect of counseling.

Hazard function analysis of relapse curves. A hazard function illustrates a different aspect of the data displayed in a relapse curve. A hazard rate is defined as the rate of relapse at a time $(t)$, given that the subjects have survived (i.e., not relapsed) to that point in time (Lee, 1992). Therefore, the hazard function provides the instantaneous relapse rate at any given time. Such an analysis is not concerned with the final abstinence rate; rather, its purpose is to reveal the underlying dynamics of the day-to-day relapse process. It serves to delineate the timely effects of counseling on the process of relapse that give rise to the differences between the final abstinence rates for the three groups.

Biomedical validation. A convenience sample of subjects who reported that they had been abstinent for at least 6 months at the time of the evaluation interview was contacted for a saliva cotinine test. Recognizing that the refusal rate for giving a saliva sample in such a lowintensity intervention was likely to be high (Glasgow et al., 1993; Velicer, Prochaska, Rossi, \& Snow, 1992), this study offered \$25 as an incentive. Of those who reported abstinence for at least 6 months, 219 $(62.9 \%)$ were asked for a saliva sample. The majority of the remaining subjects were not asked because funding for biomedical validation was no longer available; 6 were not asked because they reported that they were using nicotine gum or patches at that time. Those who were not asked for a saliva sample did nevertheless continue to receive telephone evaluation. A significant percentage of those who were asked $(44.3 \%)$ declined to provide a sample. Cotinine exceeding $10 \mathrm{ng} / \mathrm{ml}$, a level sufficient to classify a subject as a current smoker (Haley, Axelrad, \& Tilton, 1983), was present in $13.9 \%$ of the saliva samples. The high refusal rate, common for low-intensity intervention studies (e.g., $60 \%$ in Warnecke, Langenberg, Gruder, Flay, \& Leonard, 1989), makes it unreasonable to attribute all refusals to a single cause (i.e., smoking; Glasgow et al., 1993; Velicer et al., 1992). However, it is important that there be no difference in refusal rates among study groups so that the difference in their abstinence rates based on self-report cannot be attributed to a higher misreport rate in any one group. The refusal rates in this study were $48.8 \%$ for self-help, $46.4 \%$ for single counseling, and $40.4 \%$ for multiple counseling. Although the numbers suggest that those who received counseling had a lower refusal rate, the difference between groups was not statistically significant, $\chi^{2}(2, N=122)=1.06, p=.59$. The maximum percentage who could have misreported their abstinence status in each group was obtained by adding the percentage who refused the test to the percentage who had a positive cotinine test result. This generated hypothetical misreport rates of $51.3 \%$ for self-help, $54.8 \%$ for single counseling, and $50.0 \%$ for multiple counseling. Again, the difference between groups was not statistically significant $\chi^{2}(2, N=219)=$ $.42, p=.81$. Therefore, the analyses which follow were based on selfreport. However, an analysis based on the worst-case scenario is also presented.

\section{Statistical Analysis}

All the statistical tests were two-tailed tests with an alpha level of .05 , because we had a priori hypotheses about the relative differences between the three groups. Log-rank tests were used to compare the relapse curves (Lee, 1992). The Statistical Analysis System (SAS 6.08) was used (SAS Institute, Inc., 1988).

\section{Results}

\section{Overall Abstinence Rate by Intention to Treat}

Table 1 shows the percentages of subjects who abstained from smoking for different lengths of time during the 12 months since the date of screening. For every length of time considered, the counseling groups had higher abstinence rates than the self-help group. Furthermore, the longer the period under consideration, the greater was the difference among groups. For example, subjects in multiple counseling were about $25 \%$ likelier than those in self-help to quit for 1 week but nearly twice as likely to quit for 12 months.

Comparison between the groups indicates that single counseling achieved higher abstinence rates than self-help for every quitting length (all $p \mathrm{~s}<.05$ except $p=.054$ for 12 months). Multiple counseling also achieved higher abstinence rates than self-help (all ps < .005). Furthermore, multiple counseling achieved greater abstinence rates than single counseling, although the difference in the 6-month abstinence rate was not significant (and $p=.058$ for 3 months).

\section{Effect of Counseling on the 24-Hr Quit Rate in the First 3 Months}

Table 2 shows the percentage of smokers who made a serious quit attempt, defined as lasting at least $24 \mathrm{hr}$, in the first 3 months after screening. The first row gives the quit rate for those whom the helpline was able to contact. Of the subjects in the self-help group, $58.8 \%$ made at least one serious quit attempt within the first 3 months. Significantly more smokers in single counseling, $66.7 \%$, did so, $\chi^{2}(1, N=1,683)=11.07, p<.001$. A similar percentage of smokers in multiple counseling, $66.5 \%$, made a quit attempt.

The second row gives the quit rate for each group if one were to assume that none of the noncontacts made a quit attempt. Under this conservative assumption, the quit rates decreased across all three groups. Still, about half of the subjects in selfhelp made a quit attempt and both counseling groups had significantly higher quit rates than the self-help group, $\chi^{2}(1, N=$ $1,984)=5.34, p<.05$, and $\chi^{2}(1, N=1,887)=11.04, p<.001$, respectively.

\section{Effect of Counseling for Relapse Prevention}

Figure 2 shows the relapse curves for the subjects who did make a quit attempt within the first 3 months, as recorded in Table 2. These curves show that counseling helped to prevent relapse. They show, moreover, that multiple sessions prevented relapse better than a single session. The differences between groups in abstinence rates for these early quit attempts are more pronounced than the differences shown in Table 1, which are for quit attempts initiated at any time during the year after screening. Log-rank tests for the curves in Figure 2 show that the single counseling group was significantly less likely to relapse than the self-help group, $\chi^{2}(1, N=1,045)=9.03, p<$ .005 . Likewise, the multiple counseling group was significantly less likely to relapse than either the single counseling group, $\chi^{2}(1, N=1,224)=26.07, p<.0001$, or the self-help group, $\chi^{2}(1, N=1,017)=60.75, p<.0001$.

Table 3 presents additional data from these analyses of the relapse curves. The first row gives the 12-month continuous abstinence rates for those quit attempts made within the first 3 months. There is a $35 \%$ increase in the abstinence rate from 
Table 1

Overall Abstinence Rate by Intention to Treat

\begin{tabular}{ccccc}
\hline & \multicolumn{3}{c}{$\% \pm 95 \%$ confidence interval } & \\
\cline { 2 - 4 } $\begin{array}{c}\text { Length of } \\
\text { abstinence }\end{array}$ & $\begin{array}{c}\text { Self-help } \\
(n=841)\end{array}$ & $\begin{array}{c}\text { Single counseling } \\
(n=1,143)\end{array}$ & $\begin{array}{c}\text { Multiple counseling } \\
(n=1,046)\end{array}$ & $\begin{array}{c}\text { Treatment effect: } \\
\chi^{2}(2, N=3,030)\end{array}$ \\
\hline 1 week & $41.4 \pm 3.3$ & $46.5 \pm 2.9^{\mathrm{a}, \mathrm{b}}$ & $51.4 \pm 3.0$ & $19.0^{* *}$ \\
1 month & $27.0 \pm 3.0$ & $32.9 \pm 2.7^{\mathrm{a}, \mathrm{b}}$ & $38.4 \pm 2.9$ & $27.6^{* *}$ \\
3 months & $18.6 \pm 2.6$ & $23.6 \pm 2.5^{\mathrm{a}, \mathrm{b}}$ & $27.2 \pm 2.7$ & $19.3^{* *}$ \\
6 months & $12.1 \pm 2.2$ & $15.7 \pm 2.1^{*}$ & $17.0 \pm 2.3$ & $9.1^{*}$ \\
12 months & $5.4 \pm 1.5$ & $7.5 \pm 1.5^{\mathrm{a}, \mathrm{b}}$ & $9.9 \pm 1.8$ & $13.9^{* *}$ \\
\hline
\end{tabular}

Note. Subjects who could not be contacted for evaluation were considered not to have quit.

- Single counseling is significantly different from self-help (except $p=.054$ for the 12-month quit rate).

${ }^{b}$ Single counseling is significantly different from multiple counseling (except $p=.058$ for the 3-month quit rate). Multiple counseling is significantly different from self-help for all five lengths of abstinence $(p<., 005)$.

${ }^{*} p<.01$ for the overall treatment effect. ${ }^{* *} p<.001$ for the overall treatment effect.

self-help to single counseling and a nearly equal increase from single to multiple counseling.

The second row of Table 3 shows the median length of abstinence, or the time it took for half of the subjects in each group to relapse. It took only 5 days for half of the self-help group to relapse after their most recent quit attempts in the first 3 months. The median for single counseling was 11 days, twice as long as for self-help. And for multiple counseling it took over 2 months for half of the subjects to relapse, almost 6 times as long as for single counseling.

Figure 3 presents the hazard functions for the three relapse curves in Figure 2. A quick glance at these curves shows that the rate of relapse for all three groups approached the same asymptote before 1 month, with minimal fluctuations.

Figure 4 focuses on the first 30 days of the curves in Figure 3. It shows that the differences between the three groups occurred mostly within the first 2 weeks after quitting. A comparison between the self-help and multiple counseling groups shows a marked difference between the two groups at the beginning of the week, that diminished over time. By the end of the first week, the difference between the two groups was much smaller. Around 2 weeks, both curves approached the same asymptote. This same trend was true for the difference between self-help and single counseling and for that between single counseling and multiple counseling.

As a final note, we performed an analysis based on the as-

Table 2

Percentage of Quit Attempts Initiated in the First 3 Months Lasting at Least $24 \mathrm{Hr}$

\begin{tabular}{lccc}
\hline & \multicolumn{3}{c}{$\% \pm 95 \%$ confidence interval } \\
\cline { 2 - 4 } $\begin{array}{c}\text { Subjects who quit for } \\
\text { at least } 24 \mathrm{hr}\end{array}$ & Self-help & $\begin{array}{c}\text { Single } \\
\text { counseling }\end{array}$ & $\begin{array}{c}\text { Multiple } \\
\text { counseling }\end{array}$ \\
\hline $\begin{array}{c}\text { Evaluated subjects } \\
(n=2,600)\end{array}$ & $58.8 \pm 3.6$ & $66.7 \pm 3.0$ & $66.6 \pm 3.1$ \\
$\begin{array}{l}\text { All subjects, with noncontacts } \\
\text { considered as never quit } \\
(N=3,030)\end{array}$ & $50.6 \pm 3.4$ & $56.0 \pm 2.9$ & $58.4 \pm 3.0$ \\
\hline
\end{tabular}

sumption that all those who refused to provide saliva samples for cotinine testing were currently smoking, although both the literature (Glasgow et al., 1993; Velicer et al., 1992) and our experience in requesting the samples suggest that this assumption was not justified. In this worst-case scenario, about half of the subjects in each group would be considered to have misreported their abstinence status ( see Method). This produced adjusted 12 -month abstinence rates of $7.2 \%$ for self-help, $9.0 \%$ for single counseling, and $13.4 \%$ for multiple counseling. In this scenario, single counseling would no longer be significantly different from self-help, although multiple counseling would still have a significantly higher abstinence rate than self-help ( $z$ $=2.25, p<.05$ ).

\section{Discussion}

The results of this experiment indicate that telephone counseling can be an effective aid for smoking cessation. Even a single pre-quit session of telephone counseling can significantly increase both the incidence and duration of quit attempts. The results also show that a dose-response relation exists between the number of sessions offered and the duration of quit attempts. If the focus is narrowed to consider only those quit attempts initiated during the period of counseling, the effect of counseling on the abstinence rate is even more pronounced.

\section{Brief Telephone Intervention}

The fact that the single counseling group significantly outperformed the self-help group is encouraging. It is especially reassuring in view of the fact that approximately $35 \%$ of the subjects in single counseling did not receive the intended counseling. About $18 \%$ of the group either declined counseling or could not be reached by a counselor, although the evaluators later reached them. A further $17 \%$ could not be reached even by the evaluators; these were considered not to have quit. The single counseling group's improvement over the self-help group then adds to the increasing body of evidence that a well-conducted brief intervention can have a significant effect on addictive behavior change (Bien, Miller, \& Tonigan, 1993; Heather, 1989; Orleans et al., 1991). 


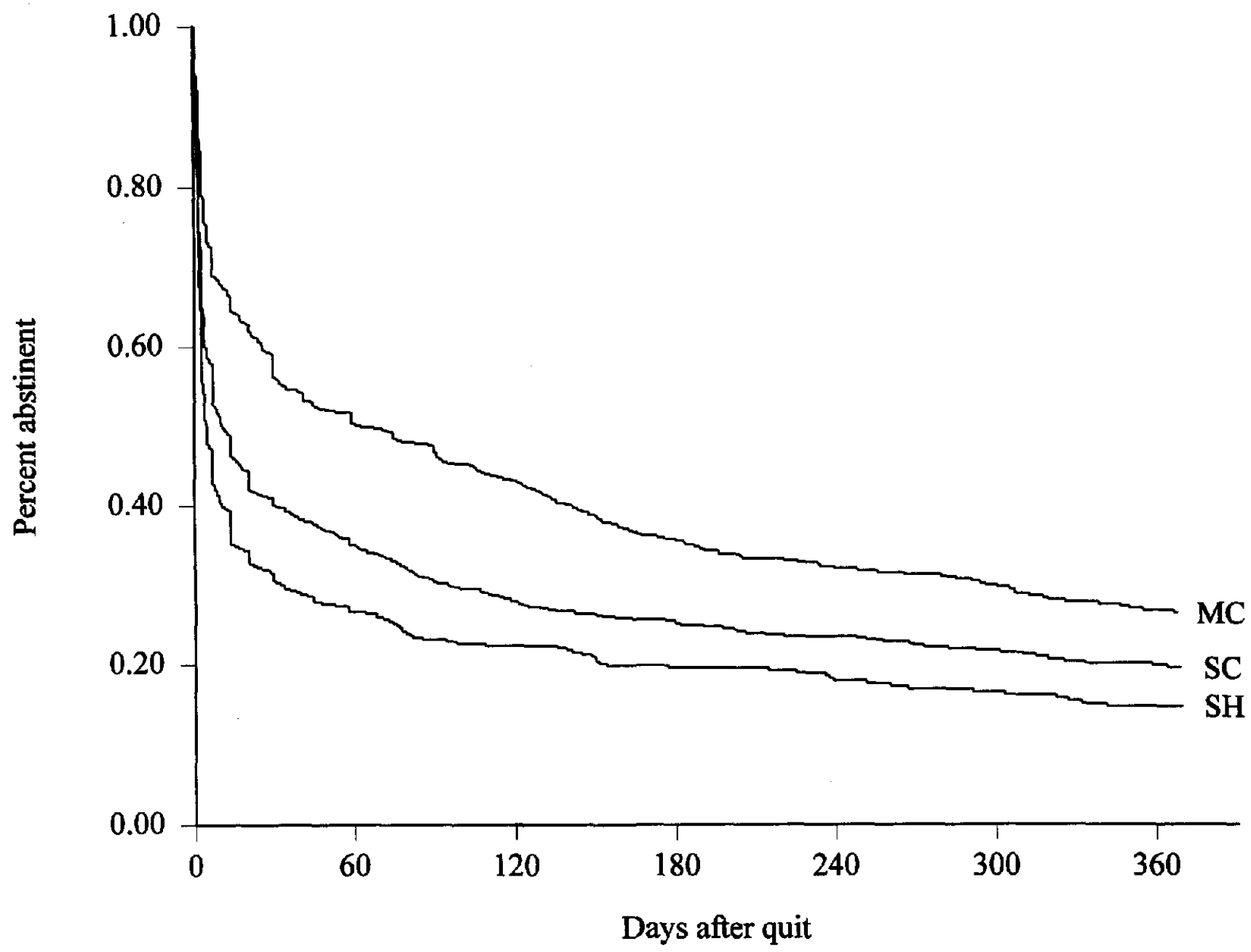

Figure 2. Relapse curves for self-help (SH), single counseling (SC), and multiple counseling (MC).

The single counseling intervention used in this study focused on two elements: promoting smokers' motivation to change (Miller \& Rollnick, 1991) and preparing them for situations in which it would be difficult to resist the urge to smoke (Marlatt \& Gordon, 1985). The first element was intended to increase the incidence of serious quit attempts; the second, to lower the relapse rate. To intervene effectively on both aspects within a limited time (about $50 \mathrm{~min}$ ) is a challenging task. For this reason, the program developed a structured protocol to ensure that clients received thorough but focused counseling (Zhu, 1993). Counseling for both motivation and relapse prevention in a single pre-quit session was clearly effective. Not only were subjects in single counseling more likely to make a quit attempt than those in self-help ( $66.7 \%$ for single counseling vs. $58.8 \%$ for selfhelp), but of subjects who quit in both groups, those in single counseling were more likely to remain abstinent for 12 months ( $19.8 \%$ vs. $14.7 \%)$.

It is worth noting, however, that many of the subjects in single counseling voiced disappointment that no follow-up counseling would be forthcoming. They seemed to think that further sessions would help them avoid relapse. Their assumption was one of the original hypotheses of the researchers and was tested in the multiple counseling group.

\section{Multisession Counseling With Relapse-Sensitive Scheduling}

A critical feature of the multiple counseling protocol of this study was the use of relapse-sensitive scheduling for follow-up sessions. A typical relapse curve, as illustrated in Figure 1, shows that the rate of relapse is very high soon after quitting and then gradually levels off. As an example, without counseling $50 \%$ of the self-help group in this study relapsed within 5 days. Because of this known pattern of relapse, the helpline scheduled three sessions in the first week and two sessions during the rest of the month.

This relapse-sensitive schedule worked well with the proactive counseling procedure, which seemed to foster both accountability and social support. Under this procedure, accountability arose from the fact that clients knew a counselor would call as scheduled to see how they were doing. At the same time, contact with a caring counselor provided social support. This 


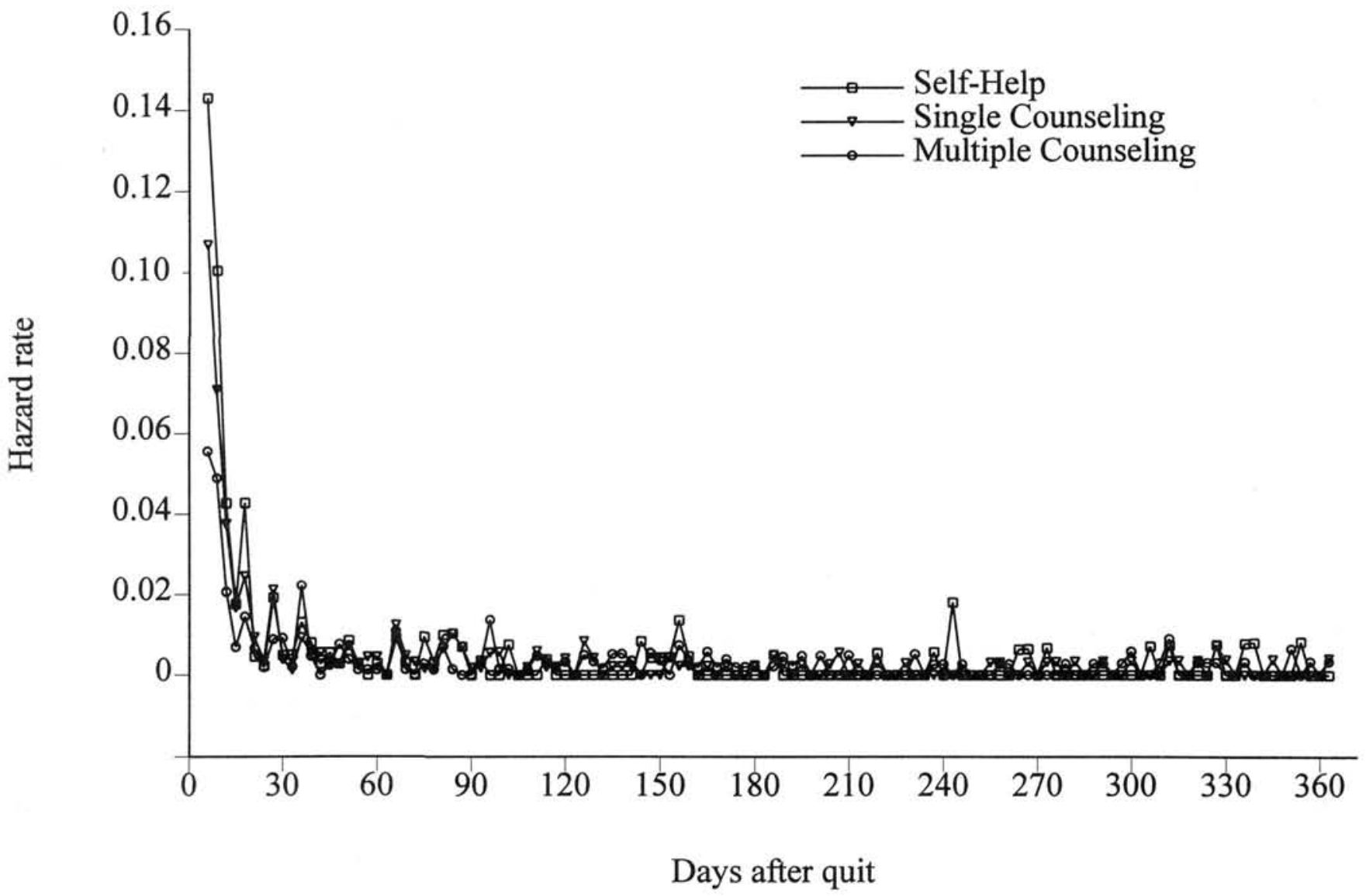

Figure 3. Hazard rates over 12 months for self-help, single counseling, and multiple counseling.

coupling of proactive counseling with relapse-sensitive scheduling provided accountability and social support when they were needed the most. Just as single-counseling subjects often yoiced disappointment that they would receive no follow-up counseling, multiple-counseling subjects frequently expressed satisfaction that they did. The sixfold difference in median quitting length between multiple and single counseling (Table 3) confirms that the proactive procedure achieved its intended effect.

The hazard function analysis presented in Figure 4 provides additional support for relapse-sensitive scheduling. These curves, depicting instantaneous relapse rates, provide a clearer picture of the time course of relapse than a typical relapse curve. The hazard function for the self-help group, for example, shows a precipitous drop in relapse probability in the first week, which suggests that intervention must occur during this period

Table 3

Twelve-Month Contimuous Abstinence Rate and Median Length of Abstinence

\begin{tabular}{|c|c|c|c|}
\hline $\begin{array}{l}\text { Rate and length of } \\
\text { abstinence }\end{array}$ & Self-help & $\begin{array}{c}\text { Single } \\
\text { counseling }\end{array}$ & $\begin{array}{l}\text { Multiple } \\
\text { counseling }\end{array}$ \\
\hline $\begin{array}{l}\text { 12-month abstinence rate } \\
\text { (\%) } \\
\text { Median length of abstinence }\end{array}$ & 14.7 & 19.8 & 26.7 \\
\hline (in days) & 5 & 11 & 63 \\
\hline
\end{tabular}

to achieve an optimal effect. Indeed, this was the period for which the majority of the follow-up sessions were scheduled for the multiple counseling group. The curve for the multiple counseling group shows that these follow-up sessions significantly lowered the relapse rate during the first week, which in turn translated into a higher 12 -month abstinence rate as shown in Figure 2.

It is worth noting that the first-week interval coincides with the peak period of self-reported withdrawal symptoms (Gross \& Stitzer, 1989; Hughes, 1990). While this does not necessarily imply a direct link between withdrawal and relapse (U.S. Department of Health and Human Services, 1990), it is conceivable that the motivation to remain abstinent would dwindle with the onset and recurrence of withdrawal symptoms. At the same time, frequent cravings for cigarettes may intensify the feeling of the need to smoke. The relapse-sensitive scheduling of follow-up sessions, therefore, addressed the issues related to withdrawal and craving in a timely manner.

Moreover, as Zhu and Pierce (1995) pointed out, the probabilistic scheduling illustrated in Figure 1 is also preferable from a learning perspective, considering that the relapse curves for smoking cessation have the same shape as typical learning curves. Accordingly, if smoking cessation were conceived of as a learning process, then the curve in Figure 1 would argue for a relapse-sensitive scheduling of follow-up sessions, regardless of the presence of withdrawal symptoms. 


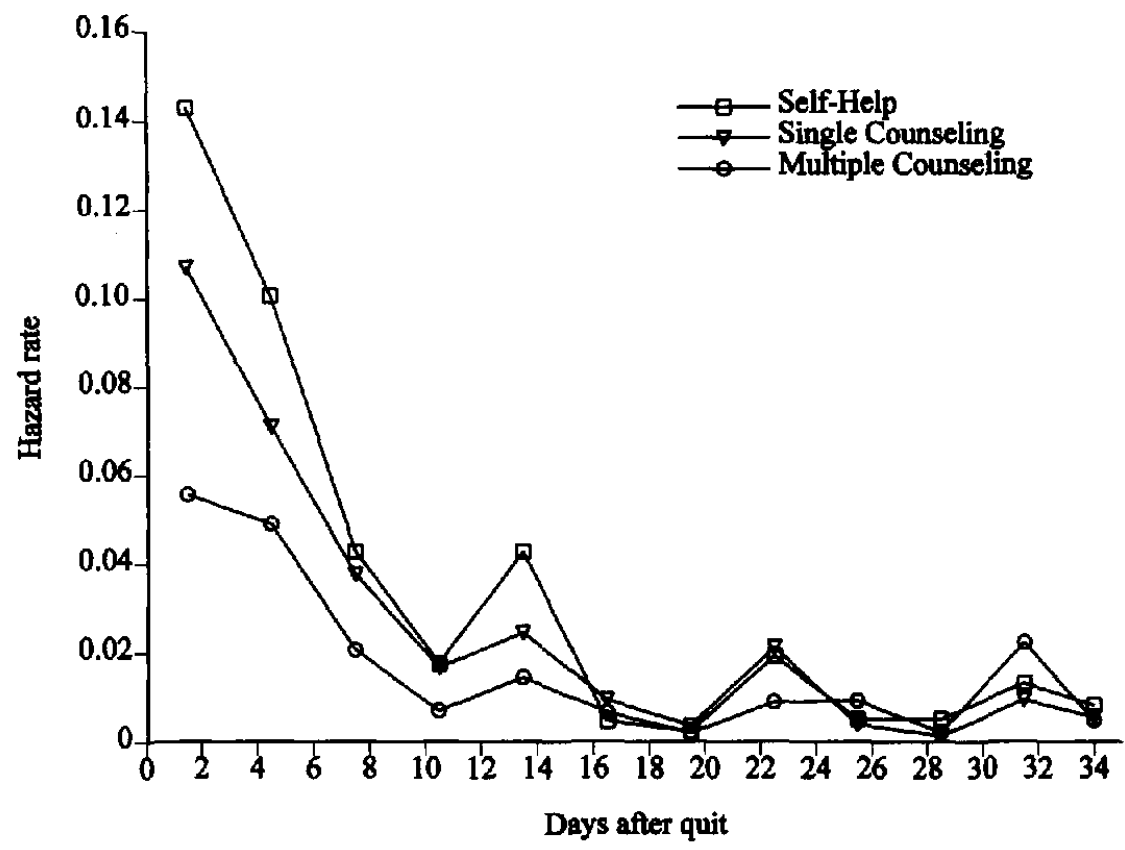

Figure 4. Hazard rates over 30 days for self-help, single counseling, and multiple counseling.

Another aspect of the pattern observed in Figures 3 and 4, which may have ramifications for the timing of brief interventions, is that the hazard curves for all three groups leveled off after 2 weeks, regardless of the intensity of intervention. This shows that those who quit smoking for 2 weeks had the same risk of relapse thereafter, regardless of group. The hazard function for the self-help group, for example, approximates the natural course of relapse. The curve for this group began to level of by the end of 1 week. Around the end of 2 weeks, the curve became asymptotic with minimal fluctuations. In other words, the risk of relapse for those who were still abstinent at 2 weeks was just as low as for those who were abstinent at 3,6 , or 9 months (Figure 3), suggesting no differential need for intervention at any time after 2 weeks for this group.

The hazard curves for the single and multiple counseling groups likewise leveled off at about 2 weeks. Before that time, as Figure 4 shows, the relapse risk was lower for single counseling than for self-help, and lower still for multiple counseling. This indicates that the pre-quit session for the single counseling group increased the number of subjects who managed to quit for 2 weeks, compared with self-help, and that the follow-up sessions in multiple counseling increased it even further. One might expect that counseling merely delayed the inevitable, that the extra subjects whom counseling enabled to quit for 2 weeks were at greater risk of relapsing later on. However, the hazard curves show that the relapse risk after 2 weeks was no greater for the counseling groups than for the self-help group. This suggests that interventions within 2 weeks of quitting can produce significant effects, but that interventions after 2 weeks ( for those who have quit) are unlikely to produce additional significant effects.

In fact, one might even gather from the data in Figure 4 that the critical period for interventions, on average, is the first week after quitting smoking. This at least was true for the majority of smokers receiving counseling from the UCSD Smokers' Helpline. Such a strong statement would be difficult to make based solely on the relapse curves in Figure 2. However, the hazard functions describing instantaneous relapse probabilities over time, in Figures 3 and 4, do suggest such an interpretation. The idea that in smoking cessation there is a critical period for the delivery of counseling services, whether 1 or 2 weeks, deserves further investigation.

\section{Dose-Response Relation}

The hypothesis that there would be a dose-response relation between the number of sessions and the effect of counseling was supported in this experiment. However, some interesting patterns emerged when the effects of multiple counseling on quit attempts and the relapse process were examined separately. The follow-up sessions given to subjects in multiple counseling had little effect on the probability of their making a quit attempt (Table 2), relative to those in single counseling. The dose-response relationship was achieved not by increasing the quit rate but by reducing the relapse rate (Figure 2 ).

The data on the rate of quit attempts in Table 2 show that one session of counseling achieved about the same effect as multiple sessions. This was somewhat unexpected because subjects in the latter group had more contact with their counselors. Specifically, subjects in single counseling neither expected nor received any follow-up sessions, even if they failed to quit on the scheduled date. In contrast, those in multiple counseling who failed to quit as planned typically received more sessions. In these sessions, their counselor would address the difficulties responsible for their failure to quit as scheduled and encourage them to set a new quit date. The counselor would then schedule the next call according to that date. For this extra effort, however, there was apparently little return. By the end of the third month, there 
was little difference in quit attempts between the two groups, as shown in Table 2.

This lack of a dose-response effect on quit attempts is to some extent consistent with the literature on brief intervention for addictive behaviors. In a recent review of interventions for problem drinking, for example, Bien et al. (1993) concluded that most studies failed to find a significant dose-response relation between the number of sessions and the treatment effect. One possible reason for this result is that the interventions studied were mostly motivational in nature; that is, once clients were motivated to change, further sessions were unlikely to produce any significant additional effect unless the added sessions changed focus (Miller \& Rollnick, 1991; W. R. Miller, personal communication, February 7,1995 ). It is obvious from the data in this experiment that continuing to counsel those who failed to quit as planned with the protocol used in this study had no immediate benefit. New strategies are needed if progress is to be made in this area.

The dose-response relation was, however, evident in the final abstinence rates in this study. In the multiple follow-up sessions responsible for this relation, the focus of counseling (for those who had made a quit attempt) changed to relapse prevention. Coping strategies discussed in the pre-quit counseling session were reviewed and their relative effectiveness evaluated. In addition, clients were urged to develop new strategies to replace ineffective ones. In later sessions, the focus shifted to self-image; that is, those who had quit were encouraged to begin seeing themselves as nonsmokers rather than as smokers who were simply not smoking. For example, they might be asked how they would respond if they were asked in a restaurant whether they wished to be seated in the smoking or nonsmoking section. Their response would then be discussed in the context of their changing self-image. Irrespective of the relative contributions of the various elements associated with the follow-up counseling (e.g., coping strategies, self-image, accountability, and social support), the relapse curves in Figure 2 show that subjects in multiple counseling were significantly less likely to relapse than those in single counseling.

It should be noted that the curve for multiple counseling in Figure 2 is a conservative estimate of the effect of the multisession counseling protocol on relapse prevention. Of the subjects in this group, $33 \%$ received no counseling, a percentage similar to that of single counseling. Of the remainder who did receive counseling, not all received the full six sessions. Some of them missed appointments or decided not to quit and dropped out early. On average, the subjects in multiple counseling received only four sessions.

The increased benefits of multiple counseling over single counseling confirm the consistency of this counseling approach. They suggest that the intervention tapped into the variables responsible for behavioral change in a predictable manner (Howard, Kopta, Krause, \& Orlinsky, 1986).

The effectiveness of the telephone counseling protocol, in combination with the high accessibility and convenience of the telephone format, suggests that this approach to smoking cessation can be used with a broader population. Currently, statewide programs modeled on this approach are in operation in California and Massachusetts.

\section{References}

Anderson, D. M., Duffy, K., Hallet, C. D., \& Marcus, A. C. (1992). Cancer prevention counseling on telephone helplines. Public Health Report, 107, 278-283.
Bien, T. H., Miller, W. R., Tonigan, J. S. (1993). Brief interventions for alcohol problems: A review. Addiction, 88, 315-336.

Curry, S. J., McBride, C. M., Louie, D., Grothaus, L. C., \& Wagner, E. H. (1992, July). Randomized trial of self-help smoking cessation intervention with non-volunteer smokers. Paper presented at the Second International Congress of Behavioral Medicine, Hamburg, Germany.

DeBusk, R. F., Miller, N. H., Superko, R., Dennis, C. A., Thomas, R. J., Lew, H. T., Berger, W. E., Heller, R. S., Rompf, J., Gee, D., Kraemer, H. C., Bandura, A., Ghandour, G., Clark, M., Shah, R., Fisher, L., \& Taylor, C. B. (1994). A case-management system for coronary risk factor modification after acute myocardial infarction. Annals of Internal Medicine, 120, 721-729.

Glasgow, R. E., Mullooly, J. P., Vogt, T. M., Stevens, V. J., Lichtenstein, E., Hollis, J. F., Lando, H. A., Severson, H. H., Pearson, K. A., \& Vogt, M. R. (1993). Biochemical validation of smoking status: Pros, cons, and data from four low-intensity intervention trials. Addictive Behaviors, 18, 511-527.

Gross, J., \& Stitzer, M. L. (1989). Nicotine replacement: Ten-week effects on tobacco withdrawal symptoms. Psychopharmacology, 98, 334-341.

Haley, N. J., Axelrad, C. M., \& Tilton, K. A. (1983). Validation of selfreported smoking behavior: Biochemical analyses of cotinine and thiocyanate. American Journal of Public Health, 38, 1204-1207.

Heather, N. (1989). Psychology and brief intervention. British Journal of Addiction, 84, 367-370.

Howard, K. I., Kopta, S. M., Krause, M. S., \& Orlinsky, D. E. (1986). The dose-effect relationship in psychotherapy. American Psychologist, 41, 159-164.

Hughes, J. R. (1990). Tobacco withdrawal in self-quitters. Journal of Consulting and Clinical Psychology, 60, 689-697.

Lando, H. A., Hellerstedt, W. L., Pirie, P. L., \& McGovern, P. G. (1992). Brief supportive telephone outreach as a recruitment and intervention strategy for smoking cessation. American Journal of Public Health, 82, 41-46.

Lee, E. T. (1992). Statistical methods for survival data analysis. New York: Wiley.

Miller, W. R., \& Rollnick, S. (1991). Motivational interviewing: Preparing people to change addictive behavior. New York: Guilford Press.

Marlatt, G. A., \& Gordon, J. R. (1985). Relapse prevention: Maintenance strategies in the treatment of addictive behavior. New York: Guilford Press.

Orleans, C. T., Schoenbach, V. J., Wagner, E. H., Quade, D., Salmon, M. A., Pearson, D. C., Fielder, J., Porter, C. Q., \& Kaplan, B. H. (1991). Self-help quit smoking intervention: Effects of self-help materials, social support intervention, and telephone counseling. Journal of Clinical and Consulting Psychology, 59, 439-448.

Ossip-Klein, D. J., Giovino, G. A., Megahed, N., Black, P. M., Emont, S. L., Stiggins, J., Shulman, E., \& Moore, L. (1991). Effects of a smokers' hotline: Results of a 10-county self-help trial. Journal of Consulting and Clinical Psychology, 59, 325-332.

Prochaska, J. O., DiClemente, C., Velicer, W. F., \& Rossi, J. (1993). Standardized, individualized interactive and personalized self-help programs for smoking cessation. Health Psychology, 12, 399-405.

SAS Institute, Inc. (1988). SAS/STAT user's guide, Release 6.08 edition. Cary, NC: Author.

Shiffman, S. (1982). Relapse following smoking cessation: A situational analysis. Journal of Consulting and Clinical Psychology, 50, 71-86.

Shiffman, S., Read, L., Maltese, J., Rapkin, D., \& Jarvik, M. E. (1985). Preventing relapse in ex-smokers: A self-management approach. In G. A. Marlatt \& J. R. Gordon (Eds.), Relapse prevention: Maintenance strategies in the treatment of addictive behavior, (pp. 472520). New York: Guilford Press.

U.S. Department of Health and Human Services. (1988). The health 
consequences of smoking: Nicotine addiction. A report of the surgeon general (DHHS Publication No. CDC 88-8416). Washington, DC: U.S. Government Printing Office.

U.S. Department of Health and Human Services. (1990). The health benefits of smoking cessation: $A$ report of the surgeon general (DHHS Publication No. CDC 90-8416). Washington, DC: U.S. Government Printing Office.

Velicer, W. F., Prochaska, J. O., Rossi, J. S., \& Snow, M. G. (1992). Assessing outcome in smoking cessation studies. Psychological Bulletin, $111,23-41$.

Warnecke, R., Langenberg, P., Gruder, C., Flay, B., \& Leonard, J. (1989). Factors in smoking cessation among participants in a televised intervention. Preventive Medicine, 18, 833-846.

Zhu, S.-H. (1993, November). Basic tenets of telephone counseling for smoking cessation. A workshop conducted at the Sixth National Conference on Nicotine Dependence, American Society of Addictive Medicine, Atlanta.

Zhu, S.-H., \& Pierce, J. P. (1995). A new scheduling method for timelimited counseling. Professional Psychology: Research and Practice, 26, 624-625.

Zhu, S.-H., Rosbrook, B., Anderson, C., Gilpin, E., Sadler, G., \& Pierce, J. P. (1995). The demographics of help-seeking for smoking cessation in California and the role of the California Smokers' Helpline. Tobacco Control, 4(Suppl. 1), S9-S15.

Received December 2, 1994

Revision received April 24, 1995

Accepted May 11, 1995 